\title{
Microvillus inclusion disease: specific diagnostic features shown by alkaline phosphatase histochemistry
}

\author{
B D LAKE
}

From the Department of Histopathology, Hospital for Sick Children and Institute of Child Health, London

SUMMARY A technique using alkaline phosphatase histochemistry on routine sections of four jejunal biopsy specimens and one necropsy sample was applied to show that alkaline phosphatase activity, normally present in the brush border, occurs in the enterocytes of patients with microvillus inclusion disease. Sections were cut at $5 \mu \mathrm{m}$, mounted on to glass slides, and dried overnight at $37^{\circ} \mathrm{C}$ before staining for alkaline phosphatase activity by the indoxyl phosphatate nitro blue tetrazolium method. Incubation periods amounted to 10 minutes for biopsy specimens and 30 minutes to one hour for necropsy samples.

The demonstration of alkaline phosphatase activity in routinely processed biopsy specimens provides an effective, quick, and definitive test in the diagnosis of microvillus inclusion disease without recourse to electron microscopy.

Microvillus inclusion disease, also known as congenital microvillous atrophy, is a disorder which presents from birth with severe intractable secretory diarrhoea.' Diagnosis is often delayed because of the difficulty in obtaining a jejunal biopsy specimen in the first weeks of life, and patients are fed intravenously until the diagnosis is made. As there is no effective treatment, accurate diagnosis is important for genetic and prognostic reasons. Histological examination shows a severe hypoplastic partial villous atrophy and an absence of the normal periodic acid Schiff (PAS) positive brush border. The apical portions, both of the surface enterocytes and some of those down the villus towards the crypt, also show PAS positive changes quite distinct from those of the goblet cells. Although this feature ${ }^{2-4}$ may be very suggestive of the diagnosis, electron microscopy is necessary to show the characteristic feature of internalised microvilli and lack or paucity of microvilli on the surface enterocytes. ${ }^{14}$ Electron microscopical examination of a suction rectal biopsy specimen may show the diagnostic changes, ${ }^{4}$ but the features are not common and may not be evident in all specimens. Suction rectal biopsy, however, is technically simpler in neonates and could provide a diagnosis if microvillus inclusion disease had been considered. In most patients the diagnosis will be made by examination of jejunal morphology in a biopsy specimen or at necropsy. Currently this

\section{Accepted for publication 17 March 1988}

examination needs to be made by electron microscopy.

This paper describes the results of a histochemical study which shows that the diagnosis can be reached at light microscopy by application of methods for demonstration of alkaline phosphatase activity in sections of routinely fixed and processed tissue.

\section{Material and methods}

Jejunal biopsy specimens taken perorally with the Watson paediatric capsule during the investigation of malabsorption, were fixed in $4 \%$ formaldehyde in phosphate buffer (buffered formalin) at room temperature and processed routinely into paraffin wax. Necropsy samples of small intestine were fixed in buffered formalin and processed routinely into paraffin wax. Jejunal biopsy specimens from four patients with microvillus inclusion disease were available as well as small intestine taken at post mortem examination in 1976 from one patient in whom the diagnosis was not made until several years later. Jejunal biopsy specimens and necropsy samples from patients without microvillus inclusion disease served as controls. Sections were cut at $5 \mu \mathrm{m}$, mounted on to glass slides, and dried overnight at $37^{\circ} \mathrm{C}$ before staining for alkaline phosphatase activity by the indoxyl phosphate-nitro blue tetrazolium method. ${ }^{5}$ The incubation medium contains $2.5 \mathrm{mg}$ p-toluidine salt of 5-bromo-4-chloro-3-indoxyl phosphate (Sigma) dissolved in $0.5 \mathrm{ml}$ dimethylformamide, $10 \mathrm{ml}$ $0.2 \mathrm{M}$ veronal acetate buffer ( $\mathrm{pH} 9 \cdot 5$ ), $5 \mathrm{mg}$ nitro blue 


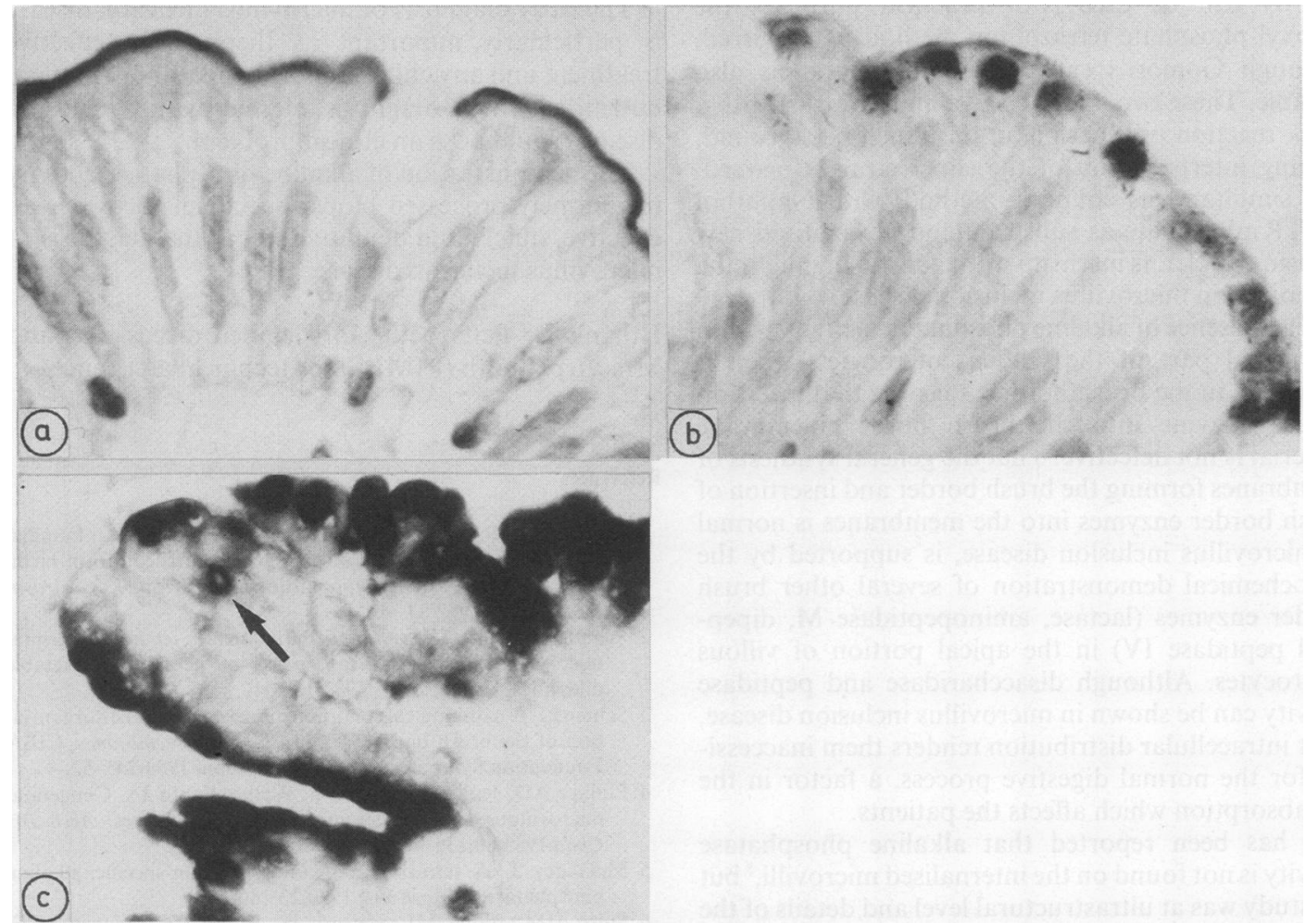

Fig 1 Jejunal biopsy specimens stained to show alkaline phosphatase activity by indoxyl phosphate-tetrazolium method. The nuclei have been counterstained with Kernechtrot. (a) normal jejunum showing discrete brush border activity; (b) microvillus inclusion disease showing clumps of activity in apical portions of enterocytes. Brush border activity is very weak.

(c) Microvillus inclusion disease with more prominent clumps of activity. Well defined ring of activity is also shown (arrow).

tetrazolium (Sigma grade III) and $0.08 \mathrm{ml} 1 \mathrm{M} \mathrm{MgCl}$. Incubation at $37^{\circ} \mathrm{C}$ for 10 minutes is required for biopsy specimens and for post mortem samples 30 minutes to one hour. Kernechtrot is a suitable nuclear counterstain.

\section{Results}

Brush border alkaline phosphatase activity (fig la) was readily demonstrable on villous enterocytes in all of the control biopsy samples which comprised those with normal morphology and those with partial villous atrophy. The activity was not present on crypt cells. A similar well defined staining pattern was found in necropsy samples which had not been autolysed.

In patients with microvillus inclusion disease alkaline phosphatase activity was present in the apical portions of the villous enterocytes. In most enterocytes the activity was present as a diffuse clump (fig 1b), but in some cells well defined ring-like activity could be seen (fig 1c). The brush border activity was severely depleted.

\section{Discussion}

The alkaline phosphatase activity of the brush border of small intestinal enterocytes is well recognised in cryostat sections of frozen tissue. Its distribution, with activity on the villous enterocyte brush border and absence within the crypts, ${ }^{6}$ serves as a useful marker in the assessment of crypt:villus ratios. Although the early studies on alkaline phosphatase activity were on tissues fixed in cold reagents for short periods of time followed by careful processing, ${ }^{7}$ it has not been widely recognised that human intestinal alkaline phosphatase is sufficiently robust to withstand routine fixation and processing. Once the tissue has been fixed and embedded in paraffin wax, alkaline phosphatase activity remains stable and can be shown in biopsy and necropsy tissue for at least 11 years. Under the same conditions the activity of other alkaline phosphatases is only poorly preserved (kidney proximal tubular enzyme) or may not be demonstrable (vascular, endothelial enzyme).

Almost any method for demonstration of enzyme 
activity can be used. For practical purposes the indoxyl phosphate-tetrazolium method ${ }^{5}$ is preferred, although Gomori's calcium-cobalt method $^{7}$ is also suitable. These two methods give high contrast with a black reaction product on an unstained background, making interpretation of the slides straightforward. The simultaneous coupling method, with Naphthol AS-TR phosphate as substrate and hexazotised new fuchsin coupler, is insensitive and fails to highlight the pathology in microvillus inclusion disease.

The presence of alkaline phosphatase activity within the apical part of the villous enterocytes, when it should be in the brush border, suggests that insertion of the enzyme into the brush border membrane material is not defective. That the general synthesis of membranes forming the brush border and insertion of brush border enzymes into the membranes is normal in microvillus inclusion disease, is supported by the histochemical demonstration of several other brush border enzymes (lactase, aminopeptidase $\mathbf{M}$, dipeptidyl peptidase IV) in the apical portion of villous enterocytes. Although disaccharidase and peptidase activity can be shown in microvillus inclusion disease, their intracellular distribution renders them inaccessible for the normal digestive process, a factor in the malabsorption which affects the patients.

It has been reported that alkaline phosphatase activity is not found on the internalised microvilli, ${ }^{8}$ but the study was at ultrastructural level and details of the methodology were not given in the summary. In our study the morphological appearances suggest that internalised microvilli (ring-like structures, fig lc) as well as the secretory granular component described at electron microscopy (diffuse clumps) both expressed alkaline phosphatase activity. Further studies on the ultrastructural localisation of alkaline phosphatase activity are planned when fresh material becomes available from this rare condition.

The pathology is consistent with the suggestion that microvillus inclusion disease is a disorder of the cytoskeleton. Depleted amounts or disordered distribution of cytoskeletal proteins, or both, have been reported for actin and cytokeratin, ${ }^{9}$ myosin, ${ }^{10}$ and vinculin." Similar ultrastructural changes can be produced experimentally in human fetal intestinal organ culture " by treatment with cytochalasins B and $D$, although there are some differences in the detailed ultrastructure. Colchicine also disturbs the cytoskeleton with formation of lateral membrane microvilli and displacement of the Golgi complex in enterocytes of the treated rat. ${ }^{12}$ Surface microvilli, however, were unaffected.
The early diagnosis of microvillus inclusion disease is particularly important as there is no effective treatment and any child supported on total parenteral nutrition with a diagnosis of microvillus inclusion disease would pose an ethical problem.

The demonstration of alkaline phosphatase activity in routinely processed biopsy specimens provides an effective, quick, and definitive test in the diagnosis of microvillus inclusion disease.

I thank Dr Peter Milla for detailed discussions and Mrs Virpi Smith (FIMLS) for technical assistance.

\section{References}

1 Davidson GP, Cutz E, Hamilton JR, Gall DG. Familia enteropathy: a syndrome of protracted diarrhoea from birth, failure to thrive and hypoplastic villus atrophy. Gastroenterology 1978;75:783-90.

2 Schmitz J, Ginies JL, Arnaud-Battandier F, et al. Congenital microvillus atrophy. A rare cause of neonatal intractable diarrhoea. Pediatric Res 1982;16:1041.

3 Schmitz J. A pathological condition due to congenital disorganisation of the brush border. In: Brush border membranes. CIBA Foundation Symposium. London: Pitman, 1983:245-52.

4 Phillips AD, Jenkins P, Raafat F, Walker-Smith JA. Congenital microvillous atrophy: specific diagnostic features. Arch Dis Child 1985;60:135-40.

5 McGadey J. A tetrazolium method for non-specific alkaline phosphatase. Histochemie 1970;23:180-4.

6 Spiro HM, Filipe MI, Stewart JS, Booth CC, Pearse AGE. Functional histochemistry of the small bowel mucosa in malabsorptive syndromes. Gut 1964;5:145-54.

7 Pearse AGE. Histochemistry-theoretical and applied. Vol 1. Third edition. London: J \& A Churchill, 1968.

8 Perez-Atayde AR, Katz A, Flores A, Madara J. A diarrheal syndrome characterised by defective assembly of absorptive cell brush borders. Lab Invest 1987;56:59A.

9 Cutz E, Durie P, Sherman P, Hamilton JR. Congenital micovillous atrophy (CMA)-disorder of enterocyte differentiation and brush border assembly. Lab Invest 1986;54:2P.

10 Carruthers L, Phillips AD, Dourmashkin R, Walker-Smith JA. Biochemical abnormality in brush border membrane protein of a patient with congenital microvillus atrophy. $J$ Pediatr Gastroenterol Nutr 1985;4:902-7.

11 Carruthers L, Dourmashkin R, Phillips AD. Disorders of the cytoskeleton of the enterocyte In: Walker-Smith JA, ed. Paediatric gastroenterology. Clinics in Gastroenterology. 1986: 105-20.

12 Pavelka M, Gangl A. Effect of colchicine on the intestinal transport of endogenous lipid. Gastroenterology 1983;84: 544-55.

Requests for reprints to: Dr B D Lake, Department of Histopathology, Hospital for Sick Children, Great Ormond Street, London WCIN 3JH, England. 